Lorena Yadira Medina Gómez

Luisa Josefina Alarcón Neve

Universidad Autónoma de Querétaro, México

\title{
El adverbio de manera corto en el español formal de México
}

Palabras clave: adverbio corto, adverbio largo, registro formal, registro informal

\section{Introducción}

En distintos dialectos del español y de otras lenguas romances se observa que los adverbios de manera cortos y largos, cuando funcionan como modificadores de predicado, tienden a ser preferidos en registros específicos. Los adverbios cortos ${ }^{1}$, derivados de las formas neutras de los adjetivos, son preferidos en registros orales-informales (directo, diario, rápido) (1); en contraste con los adverbios largos ${ }^{2}$, terminados con sufijo -mente, recurrentes en registros escritos-formales (directamente, diariamente, rápidamente) (Hummel, 2010) (2).

(1) La mujer entró directo al despacho.

(2) La mujer entró directamente al despacho.

El presente estudio se ha realizado con el fin de corroborar si en el español de México los adverbios de manera cortos con función de modificador de predicado se restringen al registro oral-informal, como muestran las tendencias de uso en otros dialectos del español (De Mello, 1992; Hummel, 2002), y en otras lenguas romances, (Hummel, 2002, 2007).

1 A lo largo de este trabajo se utilizará el término adverbio corto cuando se haga alusión al adjetivo adverbializado.

2 Se identificará al adverbio con terminación - mente como adverbio largo. 
El latín vulgar conocía el mecanismo de conversión y, muy probablemente, desconocía o usaba poco el entonces sustantivo mens bajo la forma ablativa mente para formar expresiones circunstanciales. Ello explica perfectamente el uso normal de adjetivos adverbializados en todos los estratos con tradición oral de las lenguas románicas y, como correlato, la escasez de los adverbios en -mente con función de atributos del verbo ${ }^{3}$ (Hummel, 2002: 20).

Como propósito principal se ha planteado la observación de las frecuencias de aparición de 15 pares de adverbios de manera, esto es, forma corta y larga, dentro de dos corpus de distinto registro socio-comunicativo del español de México; por un lado, el Corpus Sociolingüístico de la Cd. de México (CSCM), considerado informal por su contexto oral (3), y por el otro, el subcorpus de la Real Academia Española (CREA) limitado geográficamente al español de México y estimado como formal por sus fuentes escritas (4). En este trabajo se busca contar con evidencia suficiente para demostrar que el español de México es diferente a otros dialectos, al no usar el adverbio corto con función de modificador de predicado sólo en registros informales, sino también formales.

(3) a. [...] sea fui ese es San Luis pero pues < pus> llegamos directo [ahí es un pueblito] [...] (CSCM)

b [...] „enciende tu camioneta“/ ya la encendí y sí arrancó/ dice/ „vámonos/ vámonos/ rápido (truena los dedos)/ [luego] [...] (CSCM)

(4) a. [...] unos días más tarde, se presentó directamente en la oficina del director [...] (subcorpus CREA)

b. [...] la sacó de sus cavilaciones y la bizo buscar rápidamente una respuesta. [...] (subcorpus CREA)

\section{Los adverbios de manera en español}

La Real Academia Española RAE (2010) clasifica a los adverbios en varios tipos de acuerdo con su semántica: lugar, tiempo, cantidad, exclusión, orden, negación, afirmación, duda, adición y manera. En esta investigación sólo se considerarán los adverbios de manera, los cuales ejercen la función modificadora de predicado $(M p)^{4}$, en la que se acercan a los adjetivos (Karlsson, 1981;

3 Actualmente, el autor ha cambiado la concepción de conversión por la de atributo.

4 En este trabajo se utilizará la abreviatura $M p$ para referirse a la función de modificador de predicado. 
Hengeveld, 1992). En español han sido abordados por diversos estudios (Vigueras, 1983; Arjona, 1991; De Mello, 1992 y Hummel, 2007, 2010).

El adverbio corto tuvo su origen en el latín vulgar, su uso se limitaba al registro oral-informal. Según Karlsson (1981), el adjetivo en su forma neutra (nominativo-acusativo-neutro) cambiaba de categoría léxica gracias al proceso de conversión. Sin embargo, en estudios más recientes, Hummel (2008) prefiere el análisis que parte de proponer la existencia de una sola clase de palabra denominada atributo, en donde éste puede realizar funciones de adjetivo o de adverbio de acuerdo con la estructura sintáctica y morfológica en la que esté presente. Asimismo, Hengeveld (1992) observa que el alemán no distingue entre adjetivo y adverbio; una forma puede desempeñar ambas funciones, la adjetival y la adverbial.

Por otro lado, el adverbio largo también tiene su origen en el latín vulgar, pero se introdujo para instalarse en la norma culta; pertenece al caso ablativo de sustantivo mens que significa 'mente, espíritu, actitud'. Al inicio -mente conservó esa carga semántica, pues denotaba un estado mental determinado, bona mente, devota mente (con mente buena, hacer algo con devoción), sin embargo, con el paso del tiempo mens se desemantizó (Melis, 2006) y gramaticalizó (Magaña, 2005); de ser un lexema libre en el romance se convirtió en un morfema ligado y dejó de significar "mente" para denotar manera o modo (Karlsson, 1981; Hernández, 1992; Azpiazu, 2000; Kaul de Marlangeon, 2002; Melis, 2006 y Company Company, 2012). La distinción de uso quedó establecida: las formas terminadas en -ment fueron consideradas de uso culto, así lo corroboran textos jurídicos y eclesiásticos, "Ejemplos como bona mente factum se encuentran en textos jurídicos, [...] la opinión de Queirazza, que explica la generalización de -mente a partir de su empleo en textos católicos" (Hummel, 2002: 30-31); en contraste, las formas cortas se relacionaron con el uso vulgar.

\section{Uso de adverbios, corto y largo, en Iberoamérica y en otras lenguas romances}

Después de revisar los antecedentes históricos de estos elementos, hasta hoy día no se han encontrado análisis que comparen el comportamiento del adverbio corto y largo en el dialecto del español mexicano ni el contraste de uso de éstos en distintos registros. La presente investigación es innovadora en cuanto que observa la presencia de las dos formas de un mismo adverbio en dos registros que se diferencian entre sí por su modalidad, formal e informal; esto, para evidenciar si el adverbio corto se restringe o no a un tipo de registro. 
En cuanto al español de México existen diversos estudios que hablan del adverbio de manera en relación con su uso y clasificación. Vigueras (1983) trabaja con adverbios largos, pero no contrasta las dos formas, cortas y largas, ni observa la frecuencia de éstas conforme el nivel de registro.

Arjona (1991) describe el comportamiento sintáctico, establece una clasificación semántica de los adverbios largos en el habla popular de la Ciudad de México y compara sus resultados con los de Vigueras (1983). Al igual que Vigueras, no contrasta pares adverbiales ni considera distintos registros.

Por otro lado, De Mello (1992) estudia 189 adverbios cortos en el español culto hablado de diez ciudades. Este corpus corresponde al Proyecto de estudio coordinado de la norma lingüística culta de las principales ciudades de Iberoamérica y de la Península Ibérica5. En su análisis manifiesta que en México la frecuencia de adverbios cortos aumenta cuando hay cambio de registro, de formal a informal, y añade que el adverbio corto en el registro formal es inusual; sin embargo, esta información no puede ser determinante porque su base de datos corresponde al español oral culto. De los tres anteriores, ninguno se limita al estudio de la función de Mp.

En este tenor, Hummel (2002) ha realizado investigaciones en distintos dialectos del español de Europa y Latinoamérica sobre la preferencia de uso entre el adverbio corto y el adverbio largo con función Mp. En sus hallazgos observa preferencia del adverbio largo en contextos formales y del corto en contextos coloquiales; sin embargo, en su colecta de datos no incluye al español de México.

Por último, un estudio más reciente realizado en México por Alarcón et al. (2011), sobre el uso de adverbios cortos y largos en textos narrativos de libros de lectura utilizados oficialmente en la escuela primaria mexicana, comprobó que en 82 textos narrativos escritos por autores mexicanos hay más presencia de adverbios largos que de adverbios cortos, pero su corpus es restringido, pues sólo toma en cuenta libros de lectura de este nivel educativo; además, muestra el uso de adverbios largos y adverbios cortos en contraste con adjetivos en predicación secundaria y no considera la presencia de estos elementos en sus dos formas, lo que esta investigación pretende.

Por todo lo anterior, se considera necesario un análisis que corrobore si la forma corta de los adverbios de manera con función $M p$ se restringe o no a un registro particular en el español de México, tal y como sucede en otros

5 Las ciudades son: Bogotá, Buenos Aires, Caracas, La Habana, Lima, Madrid, México, San Juan de Puerto Rico, Santiago de Chile y Sevilla. 
dialectos. Para esto, se contrastan ambas formas adverbiales en dos corpus de distinto registro socio-comunicativo.

\section{Metodología}

En la presente investigación se trabajó con 15 adverbios, en sus dos formas, corta y larga, en dos corpus representativos y actuales; uno oral, considerado informal (CSCM), y otro escrito, considerado formal (subcorpus CREA). Estos pares adverbiales se midieron y contrastaron entre sí y entre los dos corpus para observar las tendencias de uso de cada elemento.

\subsection{Selección de datos}

Inicialmente se seleccionaron 20 adverbios analizados en tres estudios precedentes, Vigueras (1983), Arjona (1991) y Hummel (2001). De estos pares se descartaron cinco; cuatro, por tener baja frecuencia de uso en los corpus seleccionados: claro-claramente, perfecto-perfectamente, semanal-semanalmente, quincenal-quincenalmente, y uno, porque su comportamiento es principalmente como adverbio de tiempo pronto-prontamente. De esta forma, quedó una lista de 15 pares de elementos a analizar (Tabla 1 ).

\begin{tabular}{|l|l|}
\hline 1 & Rápido-rápidamente \\
\hline 2 & Diario-diariamente \\
\hline 3 & Fuerte-fuertemente \\
\hline 4 & Directo-directamente \\
\hline 5 & Fácil-fácilmente \\
\hline 6 & Lento-lentamente \\
\hline 7 & Ligero-ligeramente \\
\hline 8 & Tranquilo-tranquilamente \\
\hline 9 & Feliz-felizmente \\
\hline 10 & Bello-bellamente \\
\hline 11 & Gracioso-graciosamente \\
\hline 12 & Horrible-horriblemente \\
\hline 13 & Pobre-pobremente \\
\hline 14 & Chistoso-chistosamente \\
\hline 15 & Lindo-lindamente \\
\hline
\end{tabular}

Tabla 1. Pares de adverbios 
En el corpus del CREA, algunos adverbios fueron muy productivos como fuerte-fuertemente con 2304 ejemplos, por esta razón se decidió hacer una elección al azar de 100 elementos largos y 100 cortos dando como resultado 200 por par; lo anterior se realizó con el fin de reducirlos a un número manejable y cuidando que fuera significativo. Los elementos que no completaron 100 casos, se analizaron todos los ejemplos. Por otro lado, los ejemplos extraídos del CSCM no sufrieron este proceso, pues el par más productivo fue fácilfácilmente con 220 casos, todos los ejemplos se analizaron.

\subsection{Características del corpus del CSCM}

Este proyecto se delimitó a 27 entidades: 16 delegaciones del Distrito Federal ${ }^{6}$ y a 11 municipios conurbados del Estado de $\mathrm{México}^{7}$ a los cuales se les denominó Zona Metropolitana de la Ciudad de México (ZMCM), de acuerdo con el Censo del año 2000, 8605239 personas vivían en el Distrito Federal y 6554 239 en la zona conurbada del Estado de México (Lastra et al., 2003).

Para la elaboración del CSCM por PRESEEA-México ${ }^{8}$, se realizó un muestreo intencionado y estratificado en donde se entrevistaron a 108 personas. El tamaño de este corpus es de 901,374 palabras.

Las entrevistas realizadas se grabaron de manera individual, con una duración de entre 45 minutos a dos horas, en contextos familiares, esto es, los informantes se encontraban físicamente en su domicilio, escuela o trabajo. Esto permitió que hubiera naturalidad y espontaneidad en su oralidad, lo que observa un habla coloquial, informal. Adicional a lo anterior, es importante comentar que las producciones orales que los informantes crearon se realizaron sin una preparación o guión previo, contrario a lo que sucedería en una entrevista política, literaria, académica, etc. ${ }^{9}$ CSCM toma en cuenta tres variables sociales con valor estratificado: edad, género y grado de instrucción, con lo que se pretende tener una base de datos representativa. Todas las personas que

6 Se trata de la capital del país. Está divida en delegaciones. El proyecto eligió una variedad de éstas para tener un muestreo general.

7 El Estado de México es vecino a la capital del país y prácticamente está unido a la capital. Todos los estados de México se dividen en municipios.

8 El subcorpus PRESEEA (Proyecto para el estudio sociolingüístico del español de España y América) se desarrolla a partir de un proyecto lingüístico compartido más amplio.

9 Podría pensarse que por el hecho de ser entrevistas, los informantes tomaran una posición formal o semiformal, sin embargo, el contexto en cuanto espacio y compañía, así como las intervenciones del entrevistador menguan la formalidad que pudiera ocasionarse al inicio y terminar en un discurso relajado y confiado. 
participaron eran mayores de 20 años, hombres y mujeres nacidos en el lugar o mudados ahí desde muy pequeños. De las 108 entrevistas, las primeras 36 corresponden a un grado de instrucción bajo, de analfabetas hasta personas que terminaron la educación primaria; las siguientes 36 pertenecen al grado de instrucción intermedio, personas con secundaria o bachillerato concluido y las últimas 36, al grado alto, personas con universidad o técnica superior.

Con el propósito de crear un corpus del español urbano contemporáneo de hablantes típicos, Butragueño y Lastra (2003) en su metodología exigieron tres requisitos para ser informantes: ser parte de un núcleo urbano hispanohablante, que este núcleo hubiera estado asentado de forma tradicional en el lugar y que sus miembros presentaran heterogeneidad sociológica.

Los temas y la variedad discursiva parecen ser suficientes; los primeros se refieren a experiencias de trabajo, amigos, educación, literatura, política, tatuajes, perforaciones, familia, vacaciones, costumbres, historia, pasatiempos, etc. y los segundos albergan fragmentos expositivos, narrativos, argumentativos, descriptivos y conversacionales.

\subsection{Características del corpus del CREA}

El CREA pertenece al banco de datos de la Real Academia Española, accede a 160 millones de formas, y su estructura combina distintos criterios de selección, esto permite delimitar perfectamente lo que se quiere observar; se puede elegir una consulta especificando la distribución geográfica (país) y la distribución de medios (libros, periódicos, revistas, miscelánea y oral). En la actualidad la parte escrita del CREA cuenta con casi 5500 textos y 155 millones de formas. Con esto, el tamaño del corpus de referencia es bastante extenso para representar todas las variedades relevantes de una lengua.

Esta investigación se restringe geográficamente al subcorpus de español escrito de México, cuenta con 14 millones de palabras; sólo se toman ejemplos de los medios clasificados como libros, periódicos, revistas y miscelánea. No se tomó el medio oral ( 5 millones) porque todos los casos corresponden a contextos orales formales: entrevistas políticas, periodísticas; conferencias, contextos literarios, etc., y como se ha comentado hasta el momento, este análisis sólo se enfoca en el registro informal en lo que a oral se refiere.

Es importante resaltar que los distintos discursos de este corpus, por ser de naturaleza escrita, son preparados, revisados y reflexionados, lo que permite crear estructuras con sintaxis compleja, con mayor densidad léxica. Además, el 
objetivo principal de este tipo de discursos es exponer el material a grupos o multitudes, muchas veces especializado y crítico.

\section{Resultados}

Una vez seleccionados los ejemplos de cada corpus se descartaron los casos en los que el elemento de análisis corto funcionó como adjetivo (5), sustantivo (6) o interjección (7).

(5) La abuela cocinó rápida.

(6) ¿Sabes algo del Rápido?

(7) ¡Fácil!, ¿no?

En relación con los sustantivos e interjecciones no hubo problema en su identificación ya que su ubicación sintáctica, contenido semántico y marcas entonativas son indiscutibles; el problema se generó al diferenciar el adverbio del adjetivo porque el adjetivo masculino se traslapa con el adverbio corto con función de Mp. Para esta tarea se utilizaron las pruebas morfológicas, sintácticas y semánticas propuestas por García-Page (2002), con el fin de asegurar que estos elementos cortos funcionaran como adverbios con función de $\mathrm{Mp}$ y no como adjetivos con función de complemento predicativo (Demonte y Masullo, 1999) o como predicación secundaria (Schultze-Berndt y Himmelmann, 2004). Morfológicamente, el adverbio es invariable en género y número (8), en contraste con el adjetivo, que guarda concordancia con el sujeto (9) u objeto directo (10) de la oración.

(8) Él trabajó duro / Ella trabajó duro / Ellos trabajaron duro. [García-Page, 2002]

(9) El principe vive tranquilo / La princesa vive tranquila. / Los principes vivieron tranquilos. [García-Page, 2002]

(10) Luis compró el disco roto / Luis compró los discos rotos. [García-Page, 2002]

Sintácticamente, el adverbio ocupa un lugar prototípico dentro de la estructura, después del predicado (11), a menos que se utilicen otros recursos estilísticos para indicar alguna dislocación (12) (López, 1996: 165; Maldonado 2010:74).

(11) Él trabajó duro / * Él duro trabajó / * Duro él trabajó. [García-Page, 2002]

(12) Él trabajó duro / Él, duro, trabajó / Duro, él trabajó. [García-Page, 2002]

Por otro lado, el adjetivo goza de libertad posicional (13).

(13) El principe vive tranquilo / el principe tranquilo vive / tranquilo el principe vive. [García-Page, 2002] 
Semánticamente, el adverbio aporta matices de modo al predicado de la oración (14); sin embargo, el adjetivo con función de complemento predicativo, complementa al sujeto desde el predicado (15) (Demonte y Masullo, 1999) y con función de predicado secundario predica sobre el sujeto (16) u objeto directo (17) (Schultze-Berndt y Himmelmann, 2004).

(14) El camión avanza rápido en el salón.

(15) El agua se ve clara.

(16) Los niños van contentos al colegio.

(17) El bombre alquiló barato el departamento /alquiló barata la casa.

La complicación se aviva por las semejanzas que hay entre estas dos funciones. García-Page (2002) proporciona cuatro de ellas. Ambas construcciones responden a la pregunta ¿cómo? (18), se focalizan por medio de una oración relativa encabezada por "como" (19), se pueden sustituir por el adverbio "así" (20) y puede alternar, muchas veces, con adverbios largos (21).

(18) ¿Cómo trabajó él? [García-Page, 2002]

¿Cómo vive el príncipe? [García-Page, 2002]

(19) Duro es como trabajó él. [García-Page, 2002]

Tranquilo es como vive el príncipe. [García-Page, 2002]

(20) Él trabajó así. [García-Page, 2002]

El príncipe vive así. [García-Page, 2002]
Duro.

Tranquilo.

(21) Él trabajó duro / duramente. [García-Page, 2002]

El príncipe vive tranquilo / tranquilamente. [García-Page, 2002]

Todas estas semejanzas y diferencias se consideraron en el análisis de los casos obtenidos en ambos corpus, con el fin de seleccionar exclusivamente a los elementos adverbiales.

En el caso de los elementos largos no hubo problema, la mayoría funcionó como $\mathrm{Mp}$ (22) y en algunos casos como modificador adjetival (23).

(22) Al masticar cada fósforo cerraba los ojos fuertemente $e$ intentaba [...] CREA (23) La respiración voluntaria es fácilmente controlada por el individuo. CREA

Al concluir la depuración de los casos en donde estos elementos funcionaban como adverbios y adjetivos, se clasificaron los adverbios entre sí, distinguiendo los que ejercían la función de $\mathrm{Mp}$ de los que tenían otras funciones adverbiales sintácticas observadas ya por Vigueras (1983) y Arjona (1991): adjunto libre adverbial (24), frase adverbial (25), locución adverbial (26), modificador de cláusula (27), modificador de adjetivo (28), modificador de adverbio (29). 
(24) [...] en que una familia se acostaba, tranquilamente, con su gasto cubierto [...] CREA

(25) [...] me fui con él / así de rápido. CSCM

(26) [...] lo más natural... sucede a diario... - Señora... iPor Dios! CSCM

(27) [...] muy rápido / o sea / ella te puede ir / ir a / echarle la mano. CSCM

(28) [...] tratándose de obras directamente relacionadas CREA

(29) La figura económicamente más fuerte y desprecie al individuo [...] CREA

Al final (Tabla 2), el total de adverbios, cortos y largos, con función Mp quedó como sigue. De 2286 elementos encontrados en el CREA, 1009 funcionaron como adverbios y de esos sólo 759 como Mp. En el CSCM se hallaron 1095 casos, de ellos 372 fueron adverbios y de ese grupo 351 funcionaron como Mp.

\begin{tabular}{|l|l|l|l|}
\hline Corpus & $\begin{array}{l}\text { Total elementos } \\
\text { encontrados }\end{array}$ & $\begin{array}{l}\text { Total de ocurrencias } \\
\text { que funcionaron como } \\
\text { adverbios }\end{array}$ & $\begin{array}{l}\text { Total de ocurrencias que } \\
\text { funcionaron sólo como } \\
\text { adverbios con función Mp }\end{array}$ \\
\hline $\begin{array}{l}\text { CREA } \\
\text { (escrito) }\end{array}$ & 2286 & 1009 & 759 \\
\hline $\begin{array}{l}\text { CSCM } \\
\text { (oral) }\end{array}$ & 1095 & 372 & 351 \\
\hline
\end{tabular}

Tabla 2. Frecuencias absolutas

Debido a la reducción de los datos del CREA a un número manejable de ejemplos, no se puede comparar la aparición de los adverbios dentro de los corpus a nivel de frecuencias absolutas. Es legítimo para el análisis intra-par, pero las tendencias de uso sólo se desprenden de las frecuencias relativas, es decir, 759 ejemplos con función Mp sobre un total de 2286 palabras en el subcorpus del CREA y con el tamaño de éste, y 351 ejemplos en 1095 del CSCM con su tamaño.

La Tabla 3 muestra el resultado del análisis cuantitativo: el subcorpus del CREA muestra que de 759 (100\%) adverbios con función $M p, 74$ (10\%) son cortos y 685 (90\%) largos. La proporción se invierte respecto a lo observado en el CSCM, pues de 351 (100\%), 298 (85\%) son cortos y 53 (15\%) son largos.

Así, con base en los porcentajes de la Tabla 3, se puede establecer que la asociación entre la variable longitud del adverbio (niveles 'corto' y 'largo') está fuertemente asociada a la variable registro (niveles 'oral' y 'escrito') tal como revela el análisis estadístico: $c^{2}=103.77 ;$ g.l. $=1$ p. $<0.001$ (cálculo ajustado según el valor de corrección de Yates). 


\begin{tabular}{|l|l|l|l|}
\hline Corpus & Total adverbios Mp & Adverbios cortos Mp & Adverbios largos Mp \\
\hline $\begin{array}{l}\text { CREA / } \\
\text { escrito }\end{array}$ & $759(100 \%)$ & $74(10 \%)$ & $685(90 \%)$ \\
\hline $\begin{array}{l}\text { CSCM / } \\
\text { oral }\end{array}$ & $351(100 \%)$ & $298(85 \%)$ & $53(15 \%)$ \\
\hline
\end{tabular}

Tabla 3. Porcentaje de adverbios cortos y largos funcionando como $\mathrm{Mp}$

En la gráfica 1 se muestra la distribución general de los adverbios en ambos corpus, así como el contraste de los porcentajes generales de adverbios cortos y largos funcionando como Mp.

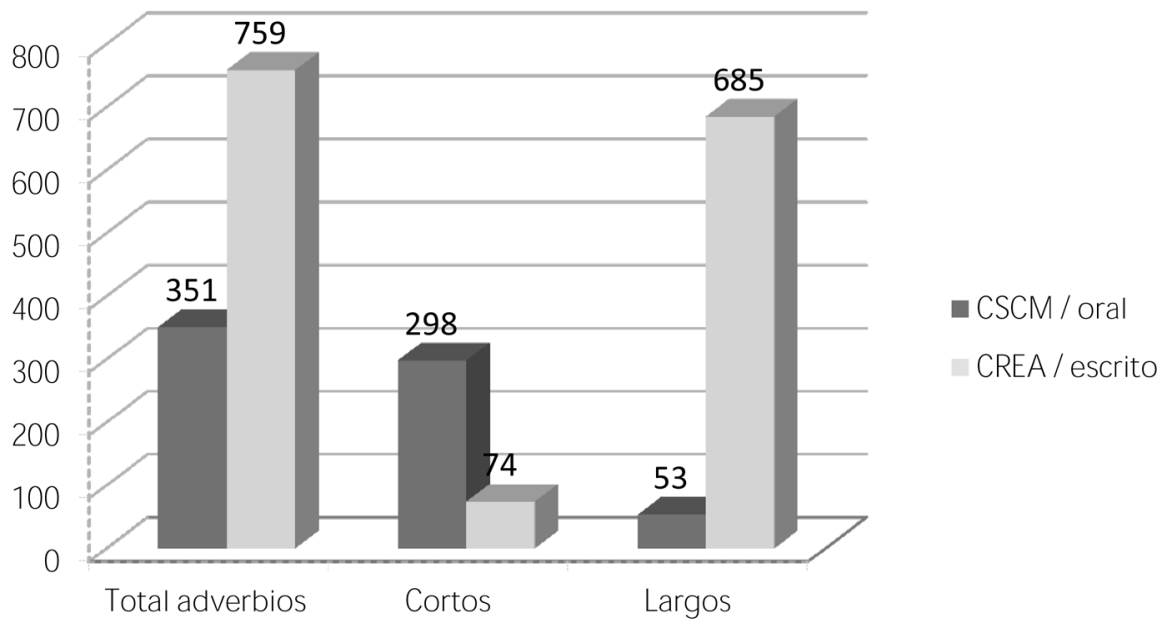

Gráfica 1. Preferencias de uso de los adverbios de manera con función Mp

Como se observa en la gráfica, el CSCM muestra significativamente más presencia de adverbios cortos que de largos, en contraste con el subcorpus del CREA del español de México, en donde las tendencias se invierten.

\subsection{Resultados del CSCM}

Se observa (Tabla 4) que los adverbios cortos extraídos del corpus CSCM tienen más frecuencia que los adverbios largos, salvo dos pares, fácil-fácilmente y directo-directamente, que tienen más recurrencia en la forma larga que en la corta, sin que altere el resultado global. Fácilmente aparece una vez más que 
fácil lo que se traduce en $51 \%$ y $49 \%$ respectivamente y directamente tres veces más que directo, lo que equivale a $54 \%$ y $46 \%$ en el mismo orden. Por otro lado, los pares que concentran la mayor muestra fueron rápido-rápidamente y diariodiariamente con 227 casos que representan el $64.6 \%$ sobre un total de 351 . Por último, los pares que no aparecieron en el CSCM fueron ligero/ligeramente, bello/bellamente, gracioso/graciosamente y lindo/lindamente.

Algunos ejemplos de adverbios cortos y largos con función de modificador de predicado, tomados de este corpus CSCM, se exhiben enseguida.

(30) a. [...] yo sí me/ aprendí un poco más rápido que mi bermano.

b. pero el bambre se/ acayó rápidamente.

(31) a. [...] ¡hijo mano/ es que la pintura es una cosa que no se da fácil!

b. [...] ay no/ tengo cada jefa// no sé decir las cosas/ no me enojo fácilmente [...]

(32) a. [...] // japonés/ cbino y todos esos/ si entras// directo/pero/y si sabes algo más de inglés/ baces un/ no/ no es cierto/ [...].

b. [...] mb// si pues sólo los que van directamente ¿no?/ y lo dejan abi [...]

(33) a. porque ya no/ ya no se puede este/viajar tranquilo en / en ningún medio.

b. y si no accedo < acedo> así tranquilamente/pues ya viene la agresión.

(34) [...] ¿.pero si yo vivo feliz en el CCH/ yo no sé (risa)/ ¿porqué ese afán de sufrir?/ ¿verdad < verdá>?"।

(35) [...] yo con mi cesárea/ ella llore y llore/ horrible/pobre de mi bija/ ¿no?/ desde abi pobre.

(36) [...][yo lo veo] que ya abi van/muy lento pero abi van.

(37) Que su mamá todo tenía que darles/ pero que este/ pues < pus> sí/ vivieron muy//pobremente/ muy bumildemente/ y<::>/ ¡cómo te diré?/ como que se bace el sufrido.

\begin{tabular}{|l|l|l|l|l|l|}
\hline \multirow{2}{*}{$\begin{array}{l}\text { Par corto-largo adverbio de } \\
\text { manera }\end{array}$} & \multirow{2}{*}{$\begin{array}{l}\text { Total de } \\
\text { adverbios }\end{array}$} & \multicolumn{4}{l}{ Adverbios como Mp } \\
\cline { 3 - 7 } & 129 & 123 & $95 \%$ & 6 & $5 \%$ \\
\hline rápido / rápidamente & 129 & 98 & $100 \%$ & 0 & $0 \%$ \\
\hline diario / diariamente & 98 & 23 & $49 \%$ & 24 & $51 \%$ \\
\hline fácil / fácilmente & 47 & 17 & $46 \%$ & 20 & $54 \%$ \\
\hline directo / directamente & 37 & 12 & $100 \%$ & 0 & $0 \%$ \\
\hline fuerte / fuertemente & 12 & 7 & $78 \%$ & 2 & $22 \%$ \\
\hline tranquilo / tranquilamente & 9 & 9 & $100 \%$ & 0 & $0 \%$ \\
\hline feliz / felizmente & 9 & & & & \\
\hline
\end{tabular}




\begin{tabular}{|l|l|l|l|l|l|}
\hline \multirow{2}{*}{$\begin{array}{l}\text { Par corto-largo adverbio de } \\
\text { manera }\end{array}$} & \multirow{2}{*}{$\begin{array}{l}\text { Total de } \\
\text { adverbios }\end{array}$} & \multicolumn{4}{l}{ Adverbios como Mp } \\
\cline { 3 - 7 } & corto & \multicolumn{2}{l}{$l \mid$} \\
\hline lento / lentamente & 4 & 4 & $100 \%$ & 0 & $0 \%$ \\
\hline pobre / pobremente & 1 & 0 & $0 \%$ & 1 & $0 \%$ \\
\hline chistoso / chistosamente & 1 & 1 & $100 \%$ & 0 & $0 \%$ \\
\hline ligero / ligeramente & 0 & 0 & $0 \%$ & 0 & $0 \%$ \\
\hline bello / bellamente & 0 & 0 & $0 \%$ & 0 & $0 \%$ \\
\hline gracioso / graciosamente & 0 & 0 & $0 \%$ & 0 & $0 \%$ \\
\hline lindo / lindamente & 0 & 0 & $0 \%$ & 0 & $0 \%$ \\
\hline Totales & 351 & $\mathbf{2 9 8}$ & & $\mathbf{5 3}$ & \\
\hline PORCENTAJE & $100 \%$ & $\mathbf{8 5} \%$ & & $\mathbf{1 5 \%}$ & \\
\hline
\end{tabular}

Tabla 4. Frecuencias de los adverbios en corpus oral (CSCM) según su función

\subsection{Resultados subcorpus CREA}

En estos resultados (Tabla 5) se observa que el adverbio largo tiene mayor presencia que el adverbio corto. Los números son contundentes de forma general, sin embargo, también hay un adverbio cuyas formas chistoso-chistosamente muestran resultados contrarios en lo particular y en este caso el resultado individual sí es representativo. Chistoso equivale al $67 \%$ y chistosamente al 33\%. Los pares más frecuentes son rápido-rápidamente, diario-diariamente, directodirectamente, fácil-fácilmente, lento-lentamente, con 495 casos lo que representa un $65.2 \%$ sobre un total de 759 . El par lindo-lindamente no presentó un solo ejemplo (véase Tabla 5).

\begin{tabular}{|l|l|l|l|l|l|}
\hline Par corto-largo adverbio de & \multirow{2}{*}{$\begin{array}{l}\text { Total de } \\
\text { manera }\end{array}$} & \multicolumn{4}{l|}{ Adverbios como Mp } \\
\cline { 3 - 7 } & & \multicolumn{2}{l}{ corto } & largo \\
\hline rápido / rápidamente & 125 & 32 & $26 \%$ & 93 & $74 \%$ \\
\hline diario / diariamente & 94 & 4 & $4 \%$ & 90 & $96 \%$ \\
\hline directo / directamente & 93 & 6 & $6 \%$ & 87 & $94 \%$ \\
\hline fácil / fácilmente & 92 & 6 & $7 \%$ & 86 & $93 \%$ \\
\hline lento / lentamente & 91 & 3 & $3 \%$ & 88 & $97 \%$ \\
\hline tranquilo/tranquilamente & 72 & 0 & $0 \%$ & 72 & $100 \%$ \\
\hline
\end{tabular}




\begin{tabular}{|l|l|l|l|l|l|}
\hline \multirow{2}{*}{$\begin{array}{l}\text { Par corto-largo adverbio de } \\
\text { manera }\end{array}$} & \multirow{2}{*}{$\begin{array}{l}\text { Total de } \\
\text { adverbios }\end{array}$} & \multicolumn{4}{l}{ Adverbios como Mp } \\
\cline { 3 - 7 } & & \multicolumn{2}{l}{ corto } & \multicolumn{2}{l}{$l$} \\
\hline fuerte / fuertemente & 59 & 11 & $19 \%$ & 48 & $81 \%$ \\
\hline feliz / felizmente & 27 & 7 & $26 \%$ & 20 & $74 \%$ \\
\hline horrible / horriblemente & 12 & 1 & $8 \%$ & 11 & $92 \%$ \\
\hline gracioso / graciosamente & 10 & 0 & $0 \%$ & 10 & $100 \%$ \\
\hline bello / bellamente & 9 & 0 & $0 \%$ & 9 & $100 \%$ \\
\hline chistoso / chistosamente & 6 & 4 & $67 \%$ & 2 & $33 \%$ \\
\hline pobre / pobremente & 2 & 0 & $0 \%$ & 2 & $100 \%$ \\
\hline lindo / lindamente & 0 & 0 & $0 \%$ & 0 & $0 \%$ \\
\hline Totales & 759 & 74 & & 685 & \\
\hline Porcentajes & $100 \%$ & $10 \%$ & & $90 \%$ & \\
\hline
\end{tabular}

Tabla 5. Frecuencias de los adverbios en corpus escrito (subcorpus del CREA) según su función

A continuación se presentan ejemplos de adverbios con función de modificador de predicado que se encontraron en esta base de datos.

(38) a. [...] la beroina matan lentamente; en este caso mataron rápido, trágicamente rápido.

b. [...] Tita se dirigió rápidamente a la puerta, la abrió y vio cómo el Pulqu ** (39) a. ¿Y con todo y lo lejos que vive viene diario a esta bora? - Vale la pena. -

b. [...] tenía que ser transportada diariamente mediante agotadores esfuerzos. (40) a. La lluvia cae fuerte.

b. [...] caminé por las avenidas abrazando fuertemente el portafolios robado.

(41) a. [...] el hombre amara como la mujer, no engañaría tan fácil como ustedes lo bacen.

b. [...] que se identifican fácilmente por el color y la manera de emplearlo.

(42) a. Y se lanza directo: [...]

b. [...] se presentó directamente en la oficina del director,

(43) a. [...] un río que fluye lento.

b. [...] sin cruzar una palabra caminaron lentamente bacia el invernadero. ${ }^{* *}$

(44) [...] bumedeció ligeramente, sobre el cuerpo de su esposo, la pechera **

(45) [...] le permitió a Castro bablar tranquilamente, durante 35 minutos, [...]

(46) a. Nació en Canadá, vivió feliz y acabó sirviendo a la ciencia en Cbile. **

b. Abora, ba logrado superar felizmente un concepto local [...] 
(47) [...] obtendrá graciosamente Su paternidad, **

(48) [...] obtenidas por sus trabajos se agotan y vive pobremente **

(49) a. [...] "El jazz no está muerto -dice Zappa- sólo buele chistoso..." [...] **

b. Al sentirse libre Palacios, saltando chistosamente, se escudó tras su auto. **

\section{Conclusiones}

A partir de los resultados obtenidos se puede establecer con evidencias cuantitativas que en el español de México hay una clara preferencia de uso por el adverbio corto de manera con función $\mathrm{Mp}$ en el registro oral-informal, lo que coincide con los hallazgos de De Mello (1992) y de Hummel (2001, 2002) respecto de las tendencias de uso. Sin embargo, se ha podido constatar que este elemento también se usa en el registro escrito-formal, si bien su frecuencia de aparición en este tipo de registro es baja. El haber encontrado ejemplos del adverbio corto en el subcorpus CREA del registro escrito-formal del español mexicano (10\%) permite afirmar que existe un uso no discriminado de este adverbio, contrario a lo que sucede en el francés de Francia y, en menor medida en el portugués de Brasil (Hummel, 2002), pues en estos últimos dialectos, el adverbio corto sufre de valor axiológico negativo por parte de los hablantes; es decir, éstos rechazan su uso en registros formales y cultos, lo consideran aberrante y vulgar; si por alguna razón se usa, se considera acto de provocación.

[...] los adjetivos adverbializados abundan en autores como Céline o Boudard, que usan el lenguaje sub-estándar. Los usan de forma conscientemente provocativa en oposición abierta al bon usage. En la literatura de argot, se nota incluso una cierta exageración en el empleo de los adjetivos adverbializados. De hecho, no refleja un uso sub-estándar real, sino una contestación provocativa de la norma culta por parte de ciertos autores (Hummel, 2002:15).

En el español de México, el adverbio corto no se percibe con esta denotación, y por ello puede ser encontrado tanto en registros informales como formales. Incluso, existen adjetivos como "feo" o "bonito" que hoy día desempeñan función de modificador de verbo como en: aquí buele feo o que te vaya bonito (Cfr. Moreno, 2003: 309-310).

Gracias al análisis cuantitativo y estadístico que se realizó aquí, se cuenta con evidencia suficiente para señalar que no hay exclusión de los adverbios cortos en el registro formal, aunque su uso manifiesta una baja frecuencia, lo que 
ubica al español de México en alineación con las tendencias generales del español y de otras lenguas romances en sus preferencias por el adverbio de manera largo, terminado en -mente, para la producción que implica mayor formalidad y el medio escrito, mientras que el adverbio de manera corto se prefiere en contextos comunicativos informales y orales.

Con esto, también se reconsidera la percepción acerca del uso mexicano del adverbio corto, estrechamente vinculado con el adjetivo (Moreno, 2003), pues aunque es altamente productivo, cuando su presencia se consulta en corpus se comprueba cuantitativamente que sigue la tendencia que De Mello (1992) y Hummel (2001, 2002) observan en sus investigaciones.

Las aportaciones del presente trabajo radican en la revisión de 15 adverbios, en sus dos formas, corta y larga, con función de modificador de predicado, observados en dos registros que se ubican en polos opuestos de formalidad y modalidad. Esto ha permitido valorar la frecuencia de una y otra formas con el fin de determinar contundentemente el uso del adverbio corto en el registro escrito-formal del español de México.

\section{Bibliografía}

Alarcón, N. L. J., Palancar E., Rodríguez I. (2011): «Semántica depictiva vs. semántica adverbial en textos narrativos de la escuela primaria». En: María del Carmen Morúa y Gerardo López Cruz (eds.), Estudios morfológicos, sintácticos, semánticos y de lingüistica aplicada, Colección Estudios Lingüísticos, 3, México: Unison, 169-191.

Arjona, I. M. (1991): Estudios sintácticos sobre el babla popular mexicana. México: UNAM.

Azpiazu, T. S. (2000): «Los adverbios en -mente en español y la formación adverbial en alemán: estudio morfológico-comparativo de esp. -mente y al. -weise». En: Contextos, XVII-XVIII/33-36, 261-277.

Company, C. C. (2012): «Condicionamientos textuales en la evolución de los adverbios en -mente». En: Revista de Filología Española, 92, 1, 9-42.

Corpus de la Real Academia Española. (2001): «Banco de datos (CREA)». Corpus de referencia del español actual: http://corpus.rae.es/creanet.html (21-03-2009).

De Mello, G. (1992): «Adjetivos adverbializados en el español culto hablado de diez ciudades». En: Lingüística del español actual, 14, 225-242. 
Demonte, V., Masullo P.J. (1999): «La predicación: los complementos predicativos». En: Ignacio Bosque, Violeta Demonte (eds.), Gramática Descriptiva de la Lengua Española. Vol. II. Madrid: Espasa, 2461-2534.

García-Page, M. (2002): «El análisis sintáctico (VII). Análisis de la oración simple. La función complemento predicativo». En: Pilar Gómez Manzano, Paloma Cuesta Martínez, Mario García-Page Sanchez, Ángeles Estévez Rodríguez (eds.), Ejercicios de gramática y de expresión (con nociones teóricas). Madrid: Centro de estudios Ramón Arces. 167-184.

Hengeveld, K. (1992): Non-verbal predication. Theory, typology, diacbrony. Berlin/New York: Mouton de Gruyter.

Hernández, C. (1992): «Contribución al estudio de los adverbios largos españoles formados con los sustantivos "guisa", "cosa" y "manera" ». En: M. Ariza, R. Cano, J. Mendoza y A. Nargona (eds.), Actas del II Congreso Internacional de Historia de la Lengua Española, Madrid: Pabellón de España, 497-504.

Hummel, M. (2001): «Adjetivos adverbales flexionados y adjetivos adverbializados invariables en castellano contemporáneo. Construcciones del tipo: los niños duermen tranquilos y María corre rápido». En: Revista Lengua. Revista de la carrera de lingüística e idiomas, 12, 9-52.

Hummel, M. (2002): «Sincronía y diacronía del sistema atributivo de las lenguas románicas». En: Revista Lengua. Revista especializada en lingüística y ciencias de lenguaje, 13, 9-48.

Hummel, M. (2007): «Adjetivos adverbializados y otros atributos directos del verbo en el habla oral informal de Chile». En: Juan Cuartero, Martine Emsel (eds.), Vernetzungen. Bedeutung in Wort, Satz und Text. Festscbrift für Gerd Wotjak zum 65 Geburtstag, Frankfurt am Main: Peter Lang, 129-149.

Hummel, M. (2008): «La predicación secundaria en el habla oral informal de Chile». En: Revista Elua. Estudios de lingüística, 22, 129-149.

Hummel, M. (2010): «La diachronie du système adverbial des langues romanes: tradition orale et tradition écrite». En: Maria Iliescu, Heidi M. SillerRunggaldier y Paul Danler (eds.), XXV Congrès International de Linguistique et Pbilologie Romanes, Innsbruck, 3-8 septembre 2007, 4, 445-461.

Karlsson, K. (1981): The evolution of MENTE in latin and romance, Tübingen: Max Niemeyer Verlag.

Kaul de Marlangeon, S. B. (2002): Los adverbios en -mente del español de boy y su función semántica de cuantificación. Madrid: Vervuert. 
Lastra, Y., Butragueño, P. M. (2003): Metodología del "proyecto para el estudio sociolingüístico del español de España y de América, México: El Colegio de México.

Lastra, Y. y Butragueño P. M. (2009): Corpus sociolingüístico de la ciudad de México. Materiales de PRESEEA - MEXICO. México: El Colegio de México.

López, E. A. (1996): La lengua coloquial de la comedia aristofánica. España: Universidad de Murcia.

Magaña, E. (2005): «El paso de dice que a dizque, de la referencia a la evidencia». En: Contribuciones desde Coatepec, 8, 59-70.

Maldonado, R. (2010): «Adjetivos en discurso: emociones, certezas, posibilidades y evidencias. Claro: de objeto perceptible a refuerzo pragmático». En: M. J. Rodríguez Espiñeira (coord.), Dialnet, España: Universidad de Santiago de Compostela.

Melis, C. (2006): «Los verbos de movimiento y la formación de los futuros perifrásticos». En: C. Company Company (coord.), Sintaxis bistórica de la lengua española, Vol. 2, España: Fondo de Cultura Económica.

Moreno de Alba, J. (2003): Suma de minucias del lenguaje. México: Fondo de Cultura Económica.

Real Academia de la Lengua Española (2010): Manual de la nueva gramática de la lengua Española. Madrid: Espasa.

Schultze-Berndt, E., Himmelmann, N. P. (2004): «Depictive secondary predicates in crosslinguistic perspective». En: Linguistic Typology, 8, 59-130.

Vigueras Á. A. (1983): «Sintaxis de los adverbios en -mente en el habla culta de la ciudad de México». En: Anuario de letras XXI. Facultad de filosofía y letras y centro de lingüística bispánica del instituto de investigaciones filológicas. México: Universidad Nacional Autónoma de México. 
Lorena Yadira Medina Gómez

Luisa Josefina Alarcón Neve

Universidad Autónoma de Querétaro, México

\section{The short adverb of manner in the formal Mexico Spanish}

Key words: short adverb, long adverb, formal register, informal register

In different Spanish dialects and in other romances languages it has been observed that the use of the short form of the adverb of manner (directo, rápido) is restricted to oral and informal registers, in contrast with the long form (directamente, rápidamente), which is preferred in writing and in formal registers. However, in Mexican Spanish, the use of the short form is more extended and it is not restricted to oral contexts. In this study we sought evidence of the use of adverbs in their short form in formal registers. To this end 15 adverbs were analyzed in their short form -adverbialzed adjectives- and long form -ending in -mente- in two corpuses of different sociocommunicative registers of Mexican Spanish.

The results show that short form adverb is also used in formal registers, but the preference for this form in oral registers and for the long form adverb in writing is confirmed. 
Lorena Yadira Medina Gómez

Luisa Josefina Alarcón Neve

Universidad Autónoma de Querétaro, México

\section{Kratka oblika načinovnega prislova v knjižni španščini Mehike}

Ključne besede: kratka oblika prislova, dolga oblika prislova, formalni jezikovni register, neformalni jezikovni register

Dialektalne različice španščine ter drugih romanskih jezikov beležijo rabo kratkega načinovnega prislova (directo, rápido) v pogovornih jezikovnih zvrsteh $\mathrm{v}$ nasprotju $\mathrm{z}$ dolgim načinovnim prislovom (directamente, rápidamente), ki se pojavlja predvsem v rabi knjižnega oz. pisnega jezika. V mehiški različici španščine pa se kratka oblika prislova razširja preko meja pogovorne rabe jezika. Prispevek poskuša predstaviti primere rabe kratkega prislova v knjižni španščini na jezikovnem gradivu, zajetem iz dveh korpusov različnih sociolongvističnih in sporočanjskih registrov mehiške španščine. Primeri obsegajo 15 kratkih oblik prislovov (t.j. pridevnikov v prislovni rabi) ter dolge oblike prislovov z obrazilom -mente. Rezultati dokazujejo, da se kratek prislov uporablja tudi $\mathrm{v}$ formalnem jezikovnem registru, $\mathrm{v}$ pogovornem jeziku pa prevladuje $\mathrm{v}$ rabi pred dolgo obliko, ki je omejena predvsem na pisno zvrst. 